\title{
Intraventricular Concealed Double Tachycardia: A Case Report
}

\author{
Shinichi Niwano, Yoshifusa Aizawa, Makoto Tamura and Akira Shibata
}

\begin{abstract}
An electrophysiologic study was performed on a patient with ischemic sustained ventricular tachycardia (VT). During pacing at the right ventricular apex, ventricular double potential was recorded at the left ventricular apex. Sustained VT was induced by double extra stimuli from the right ventricular apex. Three types of VTs with different QRS morphologies were observed, and each VT was changeable to other types. The interval between one of the two potentials and the surface QRS was constant during all VTs, but the other potential showed dissociation from the surface QRS. During this dissociation, an intrinsic rhythm of the potential was sometimes shorter than the cycle length of the VT. The mechanism of a block between the VT and a bystander could hardly explain these electrophysiologic findings. Concealed double ventricular tachycardia was considered to be the likely mechanism.
\end{abstract}

(Internal Medicine 31: 227-231, 1992)

Key words: ventricular tachycardia, electrical dissociation, electrophysiologic study

\section{Introduction}

Conduction delay in cardiac muscle may occur at the damaged area of the myocardium and it can be detected in an electrophysiological mapping study $(1-3)$. In sustained ventricular tachycardia, this area may act as a slow conduction pathway of a reentrant circuit, but may only be a bystander (4). Here, in a case with ischemic sustained ventricular tachycardia, a local electrogram from a part of the left ventricle showed dissociation from VT having its own cycle length. The cycle length of the local electrogram was somtimes shorter than that of VT. These findings may indicate intraventricular double tachycardia.

\section{Case Report}

A 77-year-old man with an inferior old myocardial infarction developed some episodes of unconsciousness. Neurological examinations were normal. During an episode of palpitation and faintness, the electrocardiogram documented sustained ventricular tachycardia (VT) with a heart rate of $192 / \mathrm{min}$, which was terminated by administration of intravenous procainamide $(200 \mathrm{mg})$. After termination of the $V^{\prime} T$, the electrocardiogram showed sinus rhythm and abnormal $Q$ waves in the leads of II, III and aVF. In a coronary arteriogram, the right coronary artery was totally occluded at its proximal site. A ventriculogram of the left ventricle showed a wall motion abnormality in the inferior and the posterior wall with an ejection fraction of $56 \%$.

Electrophysiologic study

An electrophysiologic study was performed to confirm the diagnosis of VT and to determine the drug treatment efficacy for the prevention of VT, after obtaining informed consent. Three quadripolar electrode catheters (USCI, Josephson multi-purpose catheter, 6F) were inserted into the femoral artery and vein and positioned against the right and the left ventricle and His bundle electrogram recording site. The protocol for VT induction employed programmed electrical stimulation involving single, double and triple extra stimuli and rapid stimulations from two different right ventricular sites and one left ventricular site. Extra stimuli were delivered after the patient received eight basic stimuli at two different cycle lengths (400 and $600 \mathrm{~ms}$ ); rapid stimulations were delivered at cycle lengths of 667 to $286 \mathrm{~ms}$ for 5 to $15 \mathrm{~s}$. When VT could not be induced, isoproterenol was continuously infused until the sinus rate increased by $20 \%$, and the protocol was repeated (5).

VT was not induced at the control state. But during intravenous isoproterenol infusion, non-sustained VT which lasted for 10 to $20 \mathrm{~s}$ were reproducibly induced. Intravenous administration of disopyramide of $100 \mathrm{mg}$

From The First Department of Internal Medicine, Niigata University School of Medicine, Niigata

Received for publication January 23, 1991; Accepted for publication June 13, 1991

Reprint requests should be addressed to Shinichi Niwano, MD, First Department of Internal Medicine, Niigata University School of Medicine. 1-754 Asahi-machi, Niigata 951, Japan 
failed to prevent the non-sustained VT, and ventricular double potential appeared at the left ventricular apex during VT, which was not recorded before the drug administration. The double potential was exaggerated by extra stimuli, and the interval between the stimulation artifact and the latter small part of the double potential was progressively prolonged as the coupling interval of the extra stimuli was shortened (Fig. 1). Sometimes, this small potential appeared repetitively having its own cycle length without capturing the whole ventricle (Fig. 1B). Sustained VT was induced by double extra stimuli from the right ventricular apex (Fig. 1C).

During induced sustained VT, three types of VTs with different QRS morphologies were observed and

A.

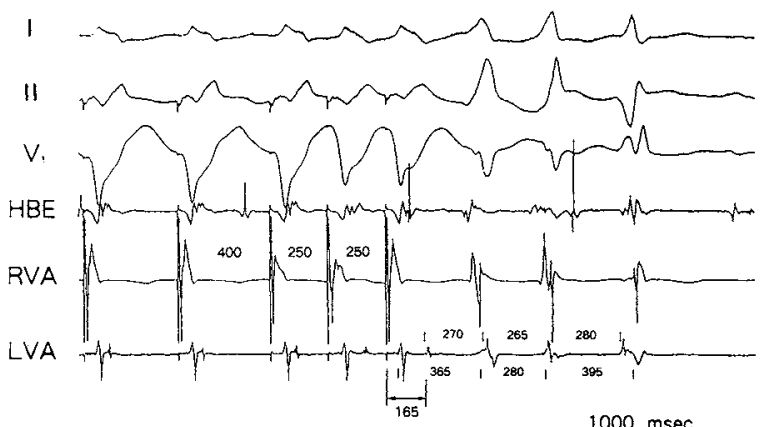

B.

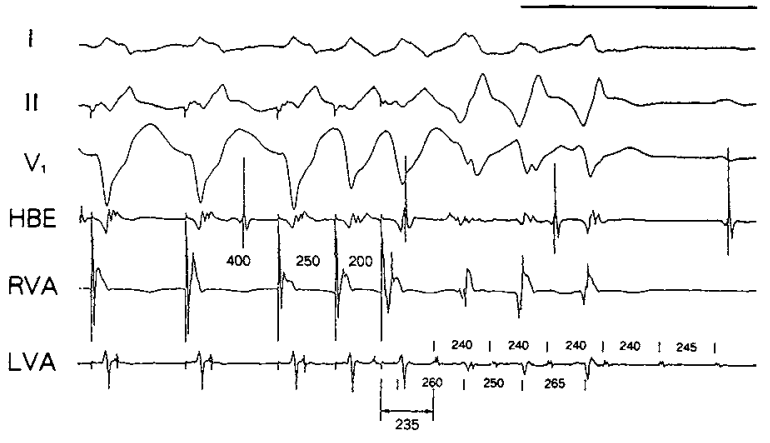

c.

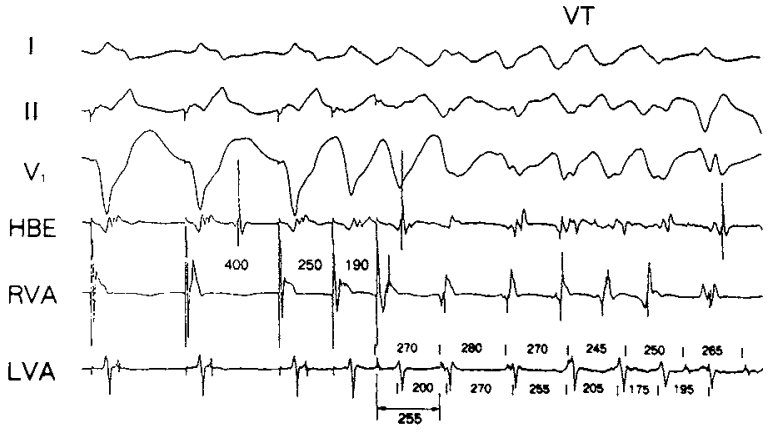

Fig. 1. Electrophysiological findings during pacing from the right ventricular apex. Panels A to $\mathrm{C}$ : Double potential was observed at the left ventricular apex during programmed stimulation. The interval between pacing artifact and the latter part of the double potential was prolonged according to the shortening of the coupling interval of extrastimuli. Panel B: A smaller potential appeared repetitively with its own cycle length without capturing the whole ventriclc. It may reflect the concealed VT near the electrode catheter. Panel C: Sustained VT was induced by double extrastimuli from the right ventricular apex. HBE: His-bundle electrogram, RVA; the right ventricular apex, LVA: the left ventricular apcx. each VT continuously changed into other VTs. The double potential was recorded at the site of the left ventricular apex during these VTs. We designated these two potentials, the large potential, L(large), and the small potential, S(small). At the time of the first VT with a normal frontal axis and right bundle branch block pattern, the time sequence of $\mathrm{L}, \mathrm{S}$ and surface QRS was constant. At the time of the second VT with right axis deviation and right bundle branch block pattern, the time sequence of $L$ and surface QRS was constant but that of $S$ was variable. The $S-S$ interval was variable, and the L-S or QRS-S interval was progressively prolonged until $S$ failed to follow them. These findings seemed to be a Wenckebach-type block-like phenomenon (Fig. 2). At the time of the third VT with right axis deviation and right bundle branch block pattern, the time sequence of $\mathrm{L}, \mathrm{S}$ and surface QRS was constant. No dissociation of any potential was observed during this type of VT morphology.

Programmed electrical stimulation of 8 basic stimuli $(220 \mathrm{~ms})$ with double extra stimuli $(210$ and $200 \mathrm{~ms})$ was delivered at the right ventricular apex during VT for termination. During pacing for the third VT, pacing captured ventricle and the QRS morphology changed gradually into a paced QRS morphology. The interval between potential $\mathrm{L}$ and the surface QRS was constant, but $\mathrm{S}$ dissociated from them and the S-S interval was constant (Fig. 3). After cessation of the pacing, QRS configuration changed into that of the first VT. Intraventricular conduction sequence was unclear; the laddergram in the figure shows one of the possibilities.

Figure 4 shows the induced non-sustained VT which included the same QRS morphology as the sustained VT. The cycle length of the VT was variable but longer than that of the sustained VT. At this time, the interval between potential $\mathrm{L}$ and the surface QRS was constant, but $\mathrm{S}$ was dissociated from the others and the S-S interval was constant and shorter than the VT cycle length.

\section{Discussion}

During the electrophysiological mapping study, ventricular delayed potential or fragmented activity may be demonstrated in a patient with sustained ventricular tachycardia $(1-4,6)$. These abnormal potentials may reflect slow conduction in the damaged myocardium (1), and the area can act in VT mechanism as a slow conduction zone of a reentrant circuit, but it may be only a bystander to the slow conduction pathway. If the area is the slow conduction zone of the reentrant circuit, the interval between the local electrogram of this site and surface QRS should be constant. If some type of exit block from the reentrant circuit exists (4), dissociation between the local electrogram and surface QRS may occur, but the cycle length of VT should be variable in this mechanism (4). If the area is only a bystander of the 


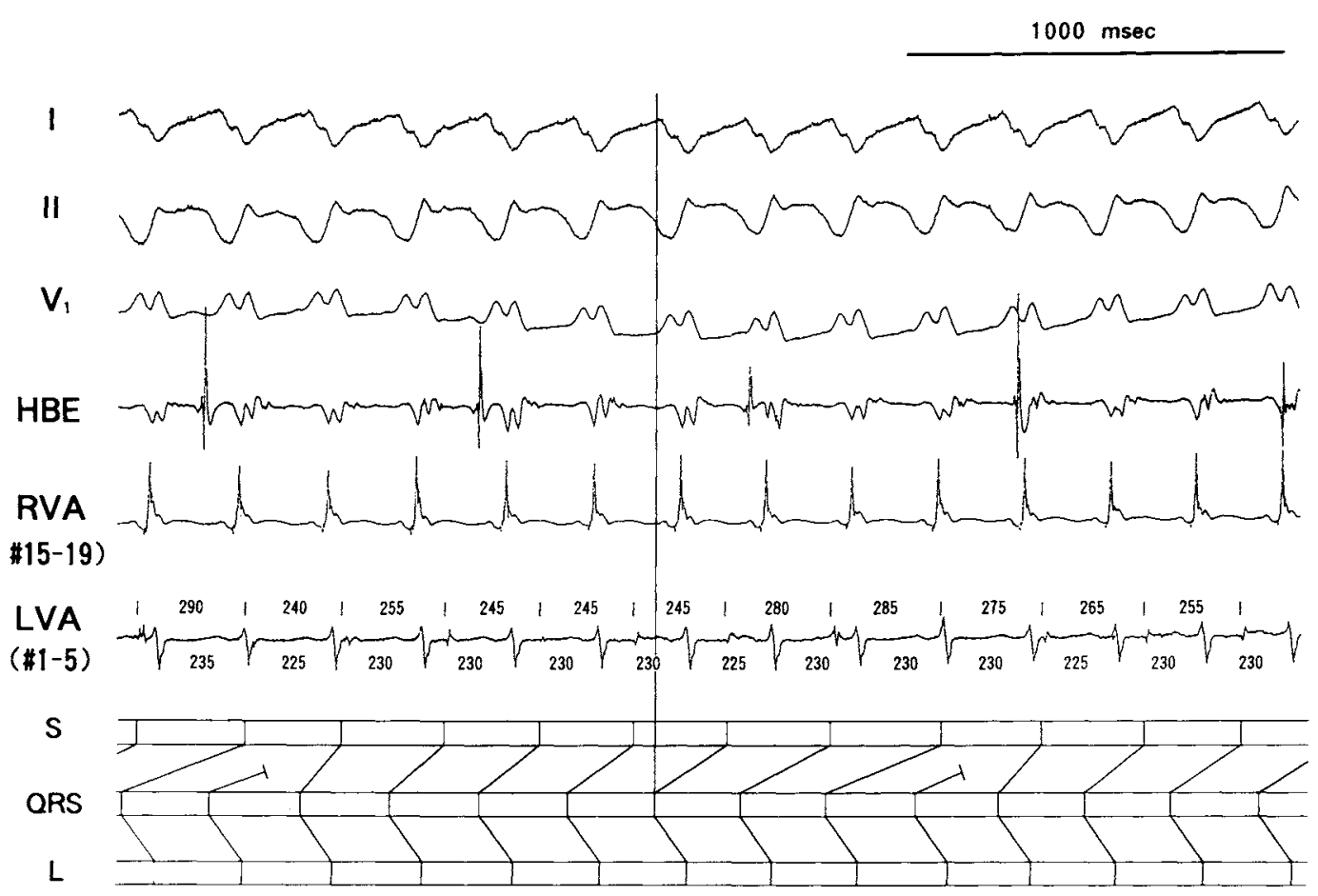

Fig. 2. Time sequence of the potentials during induced sustained VT. The second VT is shown. Potential S and VT showed a Wenckebach-type block-like finding. QRS, the onset of the surface QRS complex; L and S, The large and small parts of the double potential observed at the left ventricular apex; other abbreviations are the same as Fig. 1.

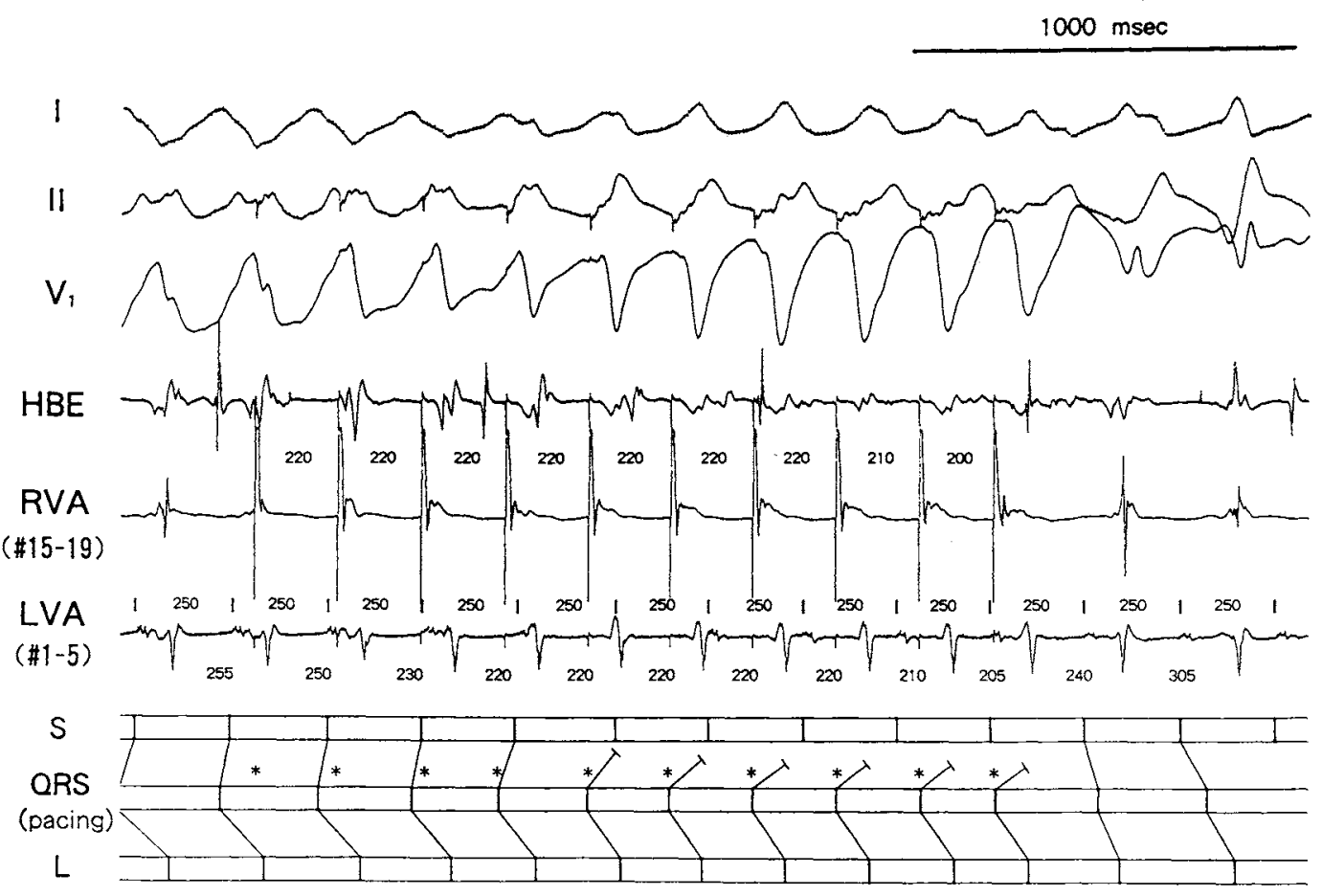

Fig. 3. Electrophysiological findings during pacing on the sustained VT. Pacing from the right ventricular apex captured the surface QRS and potential $\mathrm{L}$, but $\mathrm{S}$ dissociated and the S-S interval was constant. All abbreviations are same as Figs. 1 and 2. 

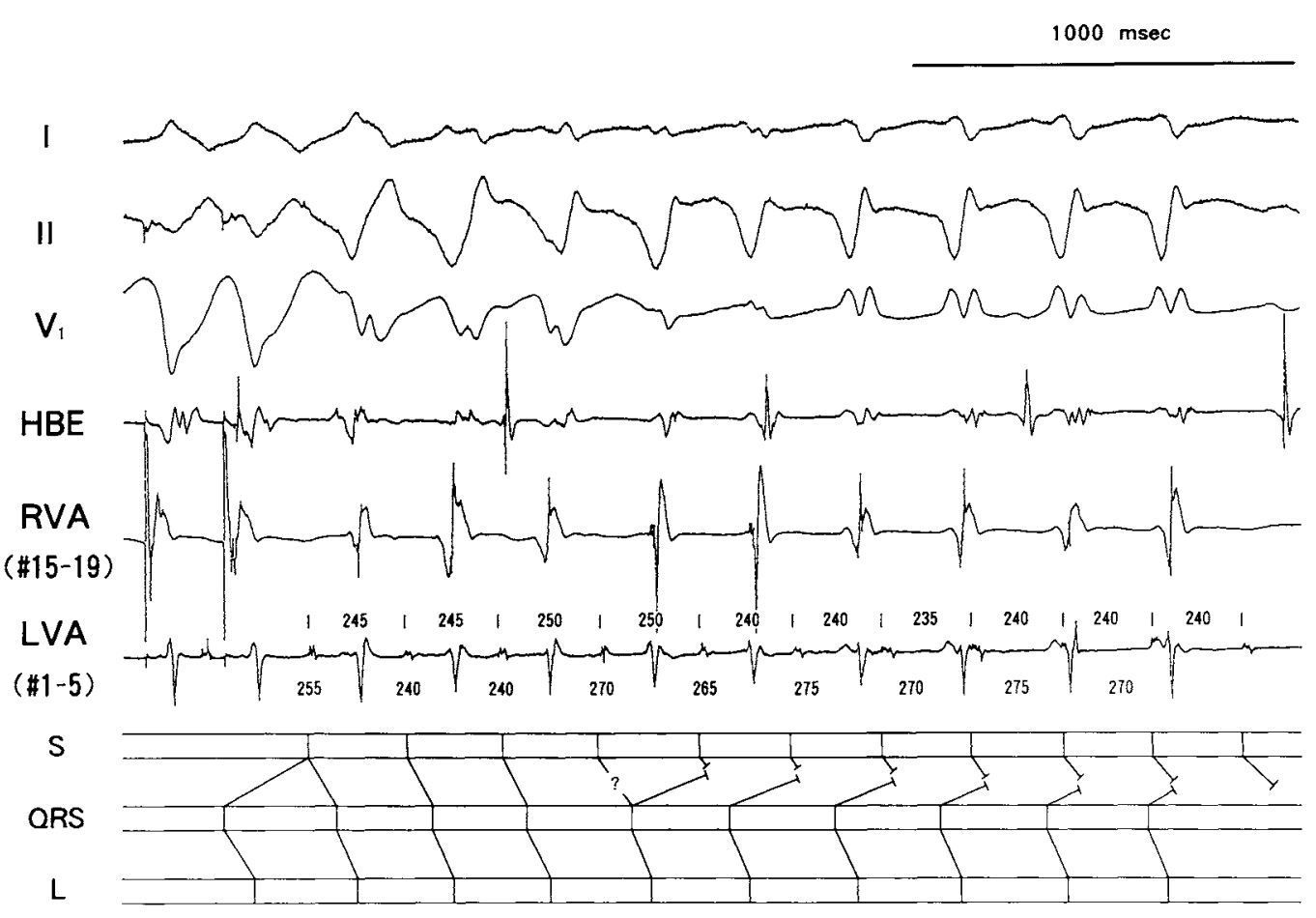

Fig. 4. Electrophysiological findings during induced non-sustained VT. The cycle length of the VT was variable. Potential S dissociated from the surface QRS and L, and the S-S interval was shorter than the cycle length of the VT. All abbreviations are the same as Figs. 1 and 2.

reentrant circuit, dissociation may also occur, but it is unusual for the local electrogram to have an intrinsic constant cycle length.

Electrophysiologic findings of this patient are summarized as follows: 1) ventricular double potential was recorded during VT at the left ventricular apex. The interval between potential $L$ and surface QRS was constant, but potential $S$ was often dissociated from the other. 2) The VT and potential $\mathrm{S}$ showed a Wenckebach type block-like pattern during the second VT, and completely dissociated with intrinsic S-S rhythm in the nonsustained VT or during pacing on VT. The intrinsic $\mathrm{S}-\mathrm{S}$ interval was longer than the R-R interval during pacing and shorter than that of non-sustained VT. 3) The potential $\mathrm{S}$ sometimes appeared repetitively with an intrinsic cycle length without capturing the whole ventricle. The findings of 2) and 3) were reproducibly observed and can hardly be explained by the mechanism of the block between the reentrant circuit and a bystander. If there is another reentrant circuit near the recording site of potential $\mathrm{S}$, these electrophysio-logical findings seem to be clearly explained.

The terms, "double tachycardia" or "simultaneous tachycardias" are defined as the simultaneous occurrence of independent atrial and ventricular (or junctional) tachycardia $(7,8)$. This type of tachycardia has been observed mainly in digitalis intoxication, generally in patients with poor left ventricular function $(8-10)$. However, other conditions with double tachycardia have been reported, such as exercise-induced double tachycardia $(11,12)$. Other investigators have reported intra atrial dissociation with simultaneous tachycardias as "atrial double tachycardia" (13). In these reports, double tachycardia was diagnosed by the recording of two dissociated and independent electrograms of each tachycardia $(7-10)$. If the interval of one potential was variable, various types of the second degree block between the two electrograms should be excluded. Then documentation of each intrinsic rhythm of each tachycardia may become strong evidence for the diagnosis of double tachycardia. In this case, potential $S$ at the left ventricular apex was mainly affected by the VT but was sometimes dissociated from the VT and showed intrinsic rhythm with a S-S interval shorter or longer than the $\mathrm{R}-\mathrm{R}$ interval. These findings indicate the existence of two simultaneous tachycardia in the ventricle. But there was no obvious evidence for the second tachycardia to make any type of VT, so the simultaneous tachycardias in this case should be termed concealed double tachycardia.

The findings of the double tachycardia or the double potential were observed only after administration of disopyramide in this case. This may mean that disopyramide had exaggerated the conduction delay in the damaged myocardium, and this effect might make the VTs inducible. However, the catheter position at the left ventricular apex could possibly have become dislocated after the drug administration in comparison with the control state, therefore the existence of the double potential at the control state cannot be denied.

It is unclear whether the potential reflects the slow 
conduction area of the reentrant circuit or only a bystander. Electrophysiological findings during rapid pacings from the site of a double potential should answer these questions $(14,15)$, but because of hemodynamic instability during VT, stimulation from the slow conduction area was not performed.

\section{Summary}

In a patient with ischemic sustained VT, ventricular double potential was recorded during VT. One of the two potentials at this site was sometimes dissociated from VT. When the potential and the VT showed a Wenckebach-type block-like pattern, the site was considered to behave as a bystander of the reentrant circuit. When the potential showed an indepandent intrinsic rhythm, intraventricular concealed double tachycardia was considered to be the mechanism.

\section{References}

1) Josephson ME, Wit AL. Fractionated electrical activity and continuous electrical activity: Fact or artifact? Circulation 70: $529,1986$.

2) Waxman HL, Sung RJ. Significance of fragmented ventricular electrograms obscrved using intracardiac recording techniques in man. Circulation 69: 1349, 1980.

3) Josephson ME, Horowitz LN, Farshidi A. Continuous local electrical activity. A mechanism of recurrent ventricular tachycardia. Circulation 57: 659, 1987.
4) Aizawa $Y$, Funazaki $T$, Satoh M, ct al. Periodic changes in cycle length of ventricular tachycardia: A Wenckebach type exit block? J Electrocardiol 19: 389, 1986.

5) Niwano S, Aizawa $Y$, Ebe $K$, et al. Efficacy and safety of ventricular pacing for ventricular tachycardia. Cardiac Pacing 4: $295,1988$.

6) Satoh M, Aizawa Y, Niwano S, ct al. Determination of the reentrant circuit by rapid pacings during ventricular tachycardia. Clin Elcctrophysiol 13: 163, 1990.

7) Bernstein LM, Pascale LR, Littman A, et al. Simultaneous independent paroxysmal tachycardias. J Am Med Assoc 150: 446, 1952.

8) Castellanos A, Azan L, Calvino JM. Simultaneous tachycardia. Am Heart J 59: 358, 1960.

9) Chowdhry IH, Hariman RJ, Gomes JA, El-Sherif N. Transient digitoxic double tachycardia. Chest 83: 686, 1983.

10) Wishner SH, Kastor JA, Yurchak PM. Double tachycardia clinical features. Circulation 38: III-217, 1969 (Abstract).

11) Eldar M, Belhassen B, Hod $\mathrm{H}$, et al. Exercise-induced double (atrial and ventricular) tachycardia: A reporte of three cases. $\mathbf{J}$ Am Coll Cardiol 14: 1376, 1989.

12) Benson DW, Gallagher JJ, Sterba R, et al. Catecholaminc induced double tachycardia: Case report in a child. PACE 3: $96,1980$.

13) Fillipi L, Alboni $P$, Malacarne $C$, et al. Double atrial tachycardia with functional atrial dissociation. J Ital Cardiol 11: 1219, 1981.

14) Morady F, Frank R, Kow W, et al. Identification and catheter ablation of a zone of slow conduction in the reentrant circuit of ventricular tachycardia in humans. J Am Coll Cardiol 11: $775,1988$.

15) Fontaine $\mathrm{G}$, Frank R, Tonct $\mathbf{J}$, Grosgogeat $\mathrm{Y}$. Identification of a zone of slow conduction. Appropriate for VT ablation: Theoretical and practical considerations. PACE 12: 262, 1989. 